\title{
Characterisation of amorphous and nanocrystalline molecular materials by total scattering $\dagger$
}

\author{
Simon J. L. Billinge, ${ }^{* a b}$ Timur Dykhne, ${ }^{a}$ Pavol Juhás, ${ }^{a}$ Emil Božin, ${ }^{a b}$ Ryan Taylor, ${ }^{c}$ Alastair J. Florence \\ and Kenneth Shankland ${ }^{d}$
}

\author{
Received 28th July 2009, Accepted 7th October 2009 \\ First published as an Advance Article on the web 19th October 2009 \\ DOI: $10.1039 / b 915453 a$
}

\begin{abstract}
The use of high-energy $X$-ray total scattering coupled with pair distribution function analysis produces unique structural fingerprints from amorphous and nanostructured phases of the pharmaceuticals carbamazepine and indomethacin. The advantages of such facilitybased experiments over laboratory-based ones are discussed and the technique is illustrated with the characterisation of a melt-quenched sample of carbamazepine as a nanocrystalline $(4.5 \mathrm{~nm}$ domain diameter) version of form III.
\end{abstract}

The majority of active pharmaceutical ingredients (APIs) are marketed as crystalline forms for reasons of stability. However, the formation, stability and performance of amorphous solids are also of significant interest within pharmaceutical research and development. Whilst the amorphous state can confer desirable properties to an API, such as increased aqueous solubility, ${ }^{1}$ the inadvertent production of non-crystalline material during processing can also lead to uncontrolled variability in physical and chemical attributes. The potential for commercial exploitation of amorphous APIs is often complicated by their tendency to revert to a more thermodynamically favourable, and less soluble, crystalline state. Although the identification, characterisation and quantification of amorphous pharmaceuticals has received considerable attention, little is known about local ordering in amorphous APIs due to the lack of reliable experimental probes. The powerful tools of crystallography begin to lose their power for structures on the nanoscale; conventional X-ray powder diffraction (XRPD) patterns become broad and featureless in these cases (Fig. 1) and are not useful for differentiating between different local molecular packing arrangements. ${ }^{2}$ Accordingly, $\mathrm{XRPD}$ is generally used simply to identify such samples as noncrystalline (i.e. 'X-ray amorphous').

It has recently been suggested that Fourier transforming conventional laboratory XRPD datat to obtain the atomic distribution function (PDF) $)^{3,4}$ allows more structural information to be extracted. ${ }^{5}$ The PDF, $G(r)$, yields the probability of finding an atom at a distance $r$ from any reference atom and so provides information on

${ }^{a}$ Department of Applied Physics and Applied Mathematics, Columbia University, New York, NY, 10027, USA. E-mail: sb2896@columbia.edu; Fax: +1 212-854-8257; Tel: +1 212-854-2918

${ }^{b}$ Condensed Matter Physics and Materials Science Department, Brookhaven National Laboratory, Upton, NY, 11973, USA

'Solid-State Research Group, Strathclyde Institute of Pharmacy and Biomedical Sciences, University of Strathclyde, Glasgow, UK G4 ONR

${ }^{d}$ School of Pharmacy, University of Reading, Reading, UK RG6 $6 A D$

$\dagger$ Electronic supplementary information (ESI) available: Sample preparation, data processing and PolySNAP comparisons of CBZ TSPDF data. See DOI: 10.1039/b915453a local structure in real space. However, this approach is intrinsically limited by the relatively low momentum transfer magnitude $Q$ $(4 \pi \sin \theta / \lambda)$ values typically accessible in the laboratory environment, resulting in a PDF of limited real-space resolution. For an accurate PDF across a wide range of $r$, data should be collected with low instrumental background and good counting statistics to high $Q$. These requirements can be met by combining high-energy (synchrotron) X-rays with imaging plate detectors.

In this work, a high-energy XRPD method known as total scattering, ${ }^{3}$ coupled with Fourier transformation and PDF analysis, is applied to individual X-ray amorphous samples $\S$ of the anti-epileptic drug carbamazepine (CBZ; Fig. 1) and the non-steroidal antiinflammatory drug indomethacin (IND). This approach is referred to as the total scattering pair distribution function (TSPDF) method to differentiate it from the approach of obtaining the PDF from conventional laboratory XRPD data. ${ }^{5}$ The TSPDF method has been widely applied to inorganic materials to study amorphous structures ${ }^{6}$ and more recently crystalline and nanocrystalline systems, ${ }^{3,7}$ but its application to molecular systems has to date been very limited.

Total scattering data were collected $\uparrow$ from melt-quenched samples of CBZ and IND as well as polycrystalline samples of CBZ I and III ${ }^{8}$ and $\alpha^{9}$ and $\gamma^{10}$ IND. The short wavelength used $(0.137 \AA$ ), combined with an appropriate data collection strategy enables data to be recorded over a sufficiently high $Q$-range to provide the necessary resolution in real-space for quantitative structural analysis to be

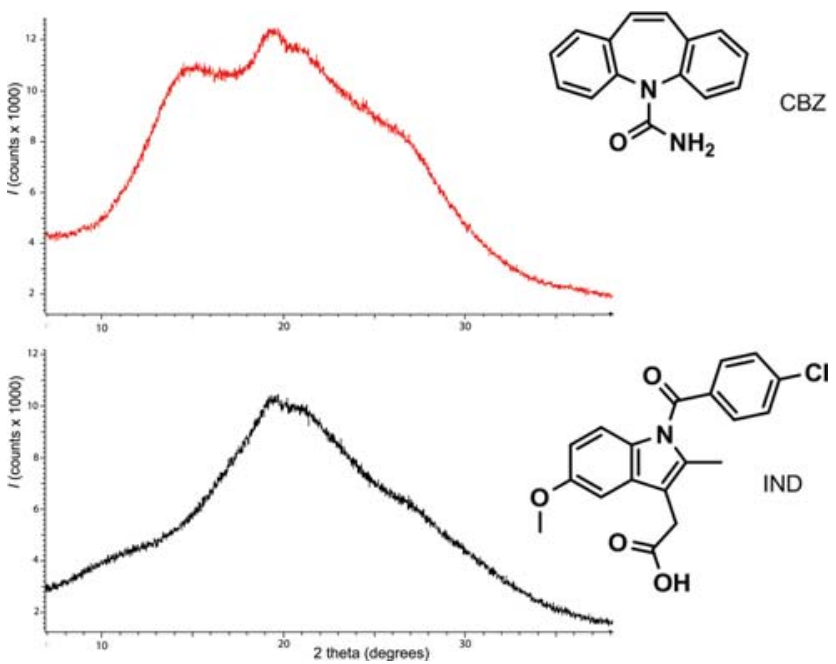

Fig. 1 Molecular structures and laboratory $\mathrm{Cu} \mathrm{K} \alpha_{1}$ XRPD patterns for $\mathrm{X}$-ray amorphous melt-quenched samples of CBZ (top) and IND (bottom). 
attempted. In these data, a useable $Q_{\max }=20 \AA^{-1}$ was achieved, equating to a real-space resolution of $0.31 \AA$. Further processing was carried out on all data sets to obtain the total scattering reduced structure function, $F(Q)$, and the TSPDF, $G(r)$, using the program PDFgetX2. ${ }^{11}$ A summary of data processing steps is provided as ESI. $\uparrow$ The total scattering data, presented as $F(Q)$, and the resultant TSPDFs for the amorphous and polycrystalline samples of CBZ are shown in Fig. 2.

$F(Q)$ for the melt-quenched sample (Fig. 2(b)), measured over a wide enough range of momentum transfer and properly normalized (see ESI) $\uparrow$ is rich in information content compared to a conventional laboratory-based XRPD measurement (e.g. Fig. 1). Close inspection shows no clear Bragg diffraction, confirming a lack of long-range order in the sample. $F(Q)$ clearly distinguishes the polycrystalline samples CBZ III and I (Fig. 2(a) and (c), respectively) with the meltquenched sample showing a closer resemblance to that of CBZ III than CBZ I.

Transforming $F(Q)$ to $G(r)$ (i.e. the TSPDF; see ESI) $\dagger$ allows interpretation and comparison to be carried out in real space. There is a striking resemblance between the TSPDF of CBZ III and the meltquenched sample. Full-profile comparisons of the TSPDFs in the range dominated by inter-molecular interactions, 3-20 А, for the three samples using PolySNAP ${ }^{12}$ yielded a correlation co-efficient of 0.8389 for the melt-quenched and CBZ III TSPDFs (perfect match $=$ 1.0 ; see ESI) $\dagger$ The next closest similarity was observed for meltquenched CBZ and form I, but yielding a correlation coefficient of only 0.5164 .

Given such close agreement between the melt-quenched and form III TSPDFs, the structural similarity between these samples was explored in more detail. The TSPDF of CBZ-III was modified by attenuating the TSPDF peaks in the high-r region to simulate the effects of reducing the range of structural coherence (or long range ordering) on the data, assuming spherical particles. If the internal atomic arrangement of a nanocrystalline domain resembles that of a bulk crystalline analog, its TSPDF resembles that of the bulk except that the amplitude of the TSPDF peaks is attenuated with increasing $r$ due to the loss of far-neighbour correlations outside the particle. This can be modelled by multiplying the crystalline PDF with the auto-correlation of the shape function of the particle as done here (see ESI) $+\dagger$
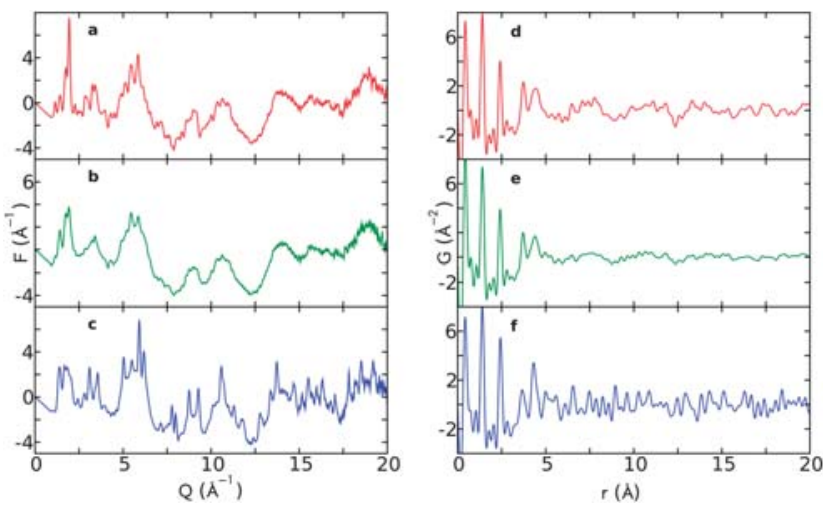

Fig. 2 Total scattering diffraction patterns and TSPDFs of CBZ. Panels (a) and (d) correspond to CBZ III, (b) and (e) to the melt-quenched sample and (c) and (f) to CBZ I; (a), (b), (c) show the total scattering data in the form of $F(Q)$ (see ESI) $\uparrow$ whilst (d), (e), (f) are in the form of the TSPDF, $G(r)$.

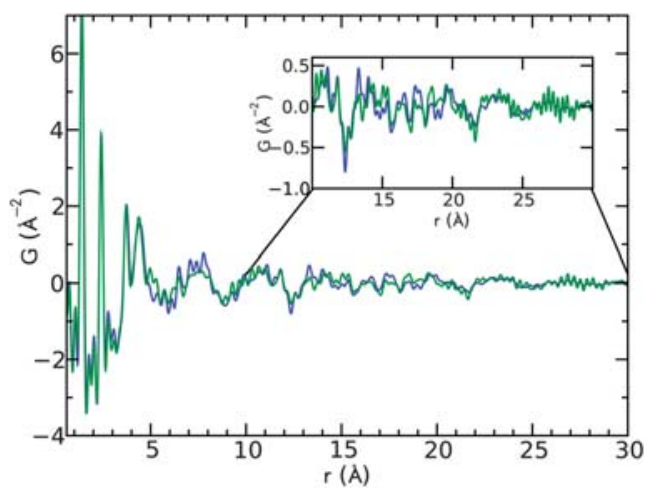

Fig. 3 Comparison of TSPDF from the melt-quenched amorphous sample (green) and CBZ III (blue), modified as if it were a $4.5 \mathrm{~nm}$ nanoparticle (see text for details). PolySNAP correlation coefficient 0.8601 .

The overlay shown in Fig. 3 was obtained using a nanocrystalline domain diameter of $4.5 \mathrm{~nm}$. The excellent agreement between the attenuated TSPDF from CBZ III and the melt-quenched CBZ TSPDF is definitive proof that the local packing in the melt-quenched sample is that of form III with a range of structural coherence of $4.5 \mathrm{~nm}$. It is interesting to ask whether the sample is made up of discrete $4.5 \mathrm{~nm}$ nanocrystallites of form III or whether it is truly a homogeneous amorphous structure with short-range molecular CBZ III-like packing. The data suggest the former since the sharpness of features in the TSPDFs is preserved with increasing $r$ whilst their amplitude is simply reduced, which is not the behaviour seen in truly amorphous samples. We thus conclude that the structure of the meltquenched CBZ used in the measurement is actually nanocrystalline CBZ III with an average particle diameter of $4.5 \mathrm{~nm}$. Although the TSPDF of the melt-quenched sample is well explained by CBZ III attenuated by the PDF characteristic function for a sphere, we cannot rule out that there is a dispersion of nanoparticle sizes centered around the value of $4.5 \mathrm{~nm}$. For example, narrow dispersions with $\sim 10 \%$ polydispersity are well explained using the characteristic function for a single sphere.

A similar analysis has also been carried out on a melt-quenched sample of IND, and the results are shown in Fig. 4. Again, $F(Q)$ shows the melt-quenched IND sample to be X-ray amorphous (no evident Bragg diffraction) and rich in structural information. The highest correlation coefficient from full-profile comparisons of the TSPDFs of melt-quenched, $\alpha$ and $\gamma$ IND in PolySNAP was 0.6770 , returned for the melt-quenched and $\alpha$-IND phases. This is significantly lower than the highest value obtained for the CBZ TSPDF comparisons. All other coefficients were less than 0.5 (ESI). $\dagger$ Thus, the TSPDFs indicate that the local structure of the melt-quenched IND sample at $100 \mathrm{~K}$ is largely distinct from the $\alpha$ and $\gamma$ crystalline forms. This contrasts with the suggestion based on crystallization and spectroscopic evidence that below $T_{\mathrm{g}}\left(315 \mathrm{~K}^{13}\right)$ amorphous IND has a local structure, with dimeric hydrogen bonding, similar to the $\gamma$ form. ${ }^{14}$ Linear combinations of the $\alpha$ and $\gamma$ crystalline phases also do not give good agreement with the TSPDF from the melt-quenched sample. Further comparisons with the $\delta$ form of IND were not possible at the time of writing as neither a crystal structure nor experimental TSPDF were available. ${ }^{15}$ However, this result clearly shows that the TSPDF can readily characterise distinct local molecular packing arrangements in the amorphous IND sample. We note 

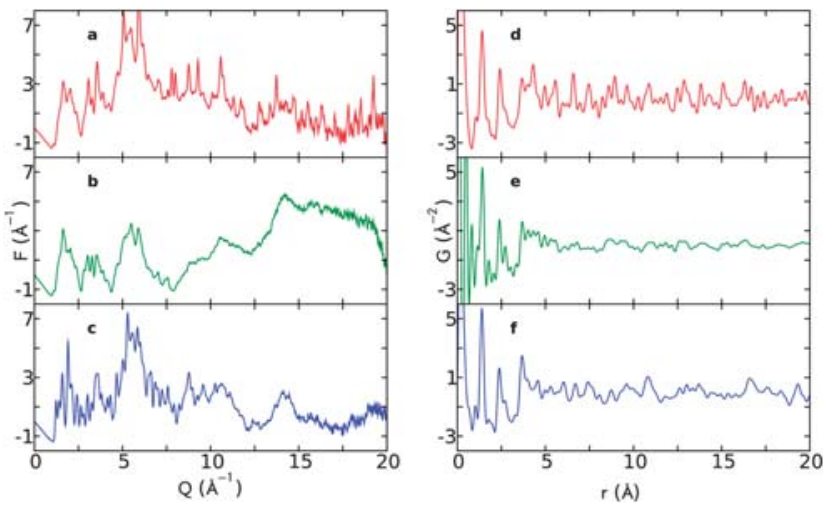

Fig. 4 Total scattering diffraction patterns and TSPDFs of IND samples. (a) and (d), contain patterns from IND $\alpha$, (b) and (e) from the melt-quenched sample and (c) and (f), IND $\gamma$. The column (a), (b), (c) shows the synchrotron total scattering data in the form of $F(Q)$ and the second column, (d), (e), (f), contains the total scattering data in the form of the TSPDF, $G(r)$.

that, as with the CBZ, oscillations in the PDF are apparent over the whole $r$-range shown and clearly extend beyond $20 \AA$, which shows that the melt-quenched IND sample we studied is also nanocrystalline rather than truly amorphous.

The key to obtaining useful TSPDF curves is not the use of synchrotron radiation per se but collecting data to high $Q$ with good statistics. This is possible from laboratory based diffractometers that have $\operatorname{Ag}(\lambda=0.556 \AA)$ or Mo $(\lambda=0.7107 \AA)$ sources, where the fact that $\lambda$ is a factor of $\sim 2-3$ times smaller than that of a $\mathrm{Cu}$ source means that higher $Q$ values can be accessed for any given diffraction angle. The current data were Fourier transformed with a $Q_{\max }$ of $20 \AA^{-1}$, which is certainly accessible with a $\mathrm{Ag}$ lab diffractometer, although a suitably configured Mo instrument would offer significant practical advantages such as higher incident flux, increased X-ray scattering and higher detector efficiency. That said, synchrotron measurements are advantageous because the requisite statistics can be obtained over the whole $Q$-range in a short time (in this case $30 \mathrm{~min}$ ) compared to many hours on a laboratory-based source. Future developments in high intensity, short wavelength laboratory X-ray sources will certainly help close this particular gap.

These results have a number of important implications. They show that TSPDF data can be used to unambiguously differentiate between different forms of amorphous or nanocrystalline molecular solids. As such, TSPDF is an approach that can take the 'fingerprinting' role for amorphous pharmaceuticals that XRPD takes for polycrystalline pharmaceuticals. This opens the door to future studies exploring the effects of processing or storage on amorphous materials and of phase stability in molecular dispersions, for example. There can also be sufficient information in the TSPDF to enable the fitting of well-defined structural models for the molecular conformation and packing arrangements in amorphous and nanocrystalline samples. Clearly this would have particular application in the case of the meltquenched IND TSPDF presented here, however the development of such models is beyond the scope of the current work. This capability offers the potential to revolutionise the study of amorphous samples, by illuminating the basic science underpinning the structure of noncrystalline molecular materials to add to the wealth of thermodynamic and spectroscopic literature available. Also, by tracking the evolution of structure of melt-quenched glasses for example, this tool may help identify new crystalline polymorphs via an amorphous or nanocrystalline route.

\section{Notes and references}

* Typically $\mathrm{Cu} \mathrm{K} \alpha$ radiation, $\lambda=1.54 \AA ; 2 \theta=60^{\circ} ; Q_{\max }=4.1 \AA^{-1}$.

$\S$ Data were collected from amorphous samples of CBZ and IND prepared by a melt-quenching method. For further details of the sample preparation see ESI. $\dagger$

I Data were collected at $100 \mathrm{~K}$ at beamline 11ID-B at the Advanced Photon Source (APS) using the rapid acquisition PDF method. Samples were sealed in $1 \mathrm{~mm}$ diameter Kapton capillaries and irradiated with X-rays of wavelength $\lambda=0.1370 \AA$. A large area $2 \mathrm{D}$ image plate detector (MAR345) was placed centered on and perpendicular to the incident beam $198 \mathrm{~mm}$ behind the sample. Sufficient statistics in the high $Q$-range were obtained using multiple exposures of the image plate, exposing for $300 \mathrm{~s}$, between 5 and 8 times for each data point. The separate exposures were summed together before further processing, resulting in an integrated exposure time of $30 \mathrm{~min}$ per sample. 1D powder diffraction patterns were obtained by integrating around the Scherrer rings on the image plate images, correcting for beam polarization effects using the program Fit2D. Data were collected to $Q=31 \mathrm{~A}^{-1}$ and Fourier transformed using data to $20 \AA^{-1}$ to generate TSPDFs to $r=30 \AA$. Further details in ESI. $\dagger$

1 B. C. Hancock and M. Parks, Pharmacol. Res., 2000, 17, 397-404.

2 S. J. L. Billinge and I. Levin, Science, 2007, 316, 561-565.

3 Takeshi Egami and S. J. L. Billinge, Underneath the Bragg peaks: structural analysis of complex materials, Pergamon, Amsterdam; London, 2003.

4 B. E. Warren, X-Ray Diffraction, Dover, New York, 1990.

5 S. Bates, G. Zografi, D. Engers, K. Morris, K. Crowley and A. Newman, Pharmacol. Res., 2006, 23, 2333-2349.

6 A. C. Wright, Phys. Chem. Glasses, 1998, 24, 148-179.

7 S. J. L. Billinge, J. Solid State Chem., 2008, 181, 1695-1700.

8 A. L. Grzesiak, M. D. Lang, K. Kim and A. J. Matzger, J. Pharm. Sci., 2003, 92, 2260-2271.

9 X. M. Chen, K. R. Morris, U. J. Griesser, S. R. Byrn and J. G. Stowell, J. Am. Chem. Soc., 2002, 124, 15012-15019.

10 P. J. Cox and P. L. Manson, Acta Crystallogr., Sect. E: Struct. Rep. Online, 2003, 59, o986-O988.

11 Xiangyun Qiu, Jeroen W. Thompson and Simon J. L. Billinge, J. Appl. Crystallogr., 2004, 37, 678.

12 G. Barr, W. Dong and C. J. Gilmore, J. Appl. Crystallogr., 2004, 37, 658-664.

13 V. Andronis and G. Zografi, J. Non-Cryst. Solids, 2000, 271, 236-248.

14 E. Y. Shalaev and G. Zografi, in Amorphous Food and Pharmaceutical Systems, ed. Harry Ed Levine, Royal Society of Chemistry, Cambridge, 2002, pp. 11-30.

15 L. Borka, Acta Pharma. Suec., 1974, 11, 295-303. 